\title{
Influência do método de vulcanização nas propriedades mecânicas e na densidade de ligações cruzadas da borracha natural
}

\section{The influence of vulcanization method on mechanical properties and crosslink density of natural rubber}

\author{
Maria Aparecida de Souza Oliveira ${ }^{1,2 *}$, Silvana Navarro Cassu ${ }^{1,2}$, Sandra Aparecida Coelho de Mello² e \\ Jorge Carlos Narciso Dutra ${ }^{1}$
}
'Departamento de Química, Instituto Tecnológico de Aeronáutica - ITA, São José dos Campos, SP, Brasil ${ }^{2}$ Divisão de Química, Instituto de Aeronáutica e Espaço - IAE, São José dos Campos, SP, Brasil
*mariamaso@iae.cta.br

\begin{abstract}
Resumo
A vulcanização é um importante fator que determina as propriedades mecânicas da borracha, mas a influência do método de vulcanização nas propriedades mecânicas do material, ainda é um assunto pouco explorado na literatura. Neste artigo, uma formulação à base de poli-cis-isopreno ou borracha natural (NR), denominada NR4010-A, foi vulcanizada em prensa e autoclave, com o objetivo de avaliar a influência do método de vulcanização na formação das ligações cruzadas e, consequentemente, nas propriedades mecânicas do material. Amostras do material vulcanizado, pelos dois métodos, foram caracterizadas por meio de análise reométrica, ensaio de inchamento e testes mecânicos, onde foi avaliada a resistência ao rasgo e à tração. Os resultados mostraram que para essa formulação, nas mesmas condições de temperatura, o método de vulcanização por autoclave se mostrou mais eficiente em relação às propriedades estudadas.
\end{abstract}

Palavras-chave: ligações cruzadas, NR, propriedades mecânicas, vulcanização.

\begin{abstract}
Vulcanization is an important factor on rubber mechanical properties determination. The influence of vulcanization method on mechanical properties is still an ongoing issue. Thus, a composition based in natural rubber (NR), named NR4010-A, was vulcanized by autoclave and press, on purpose to evaluated the vulcanization method influence on crosslink formation and thereafter on the mechanical properties of this material. Samples of vulcanized material in both conditions were characterized through swelling, rheometer tests, tear strength, strain strength and hardness. The mechanical properties studied and crosslink density were increased when the material was vulcanized by autoclave. Therefore, autoclave vulcanization method showed be more effective than press at the same conditions of temperature.
\end{abstract}

Keywords: crosslink, NR, mechanical properties, vulcanization.

\section{Introdução}

Durante a etapa de desenvolvimento de uma formulação elastomérica, a borracha submetida a uma determinada temperatura, passa por mudanças físicas e químicas que levam à alteração de suas propriedades, devido à formação de ligações cruzadas entre as cadeias poliméricas. Essas alterações se devem a fatores como: método de vulcanização, tipo de elastômero, presença de aditivos (ativadores, agentes de vulcanização), tempo e temperatura de vulcanização ${ }^{[1]}$.

Os sistemas de vulcanização mais comuns na indústria são os que utilizam enxofre ou peróxido. No sistema de vulcanização por enxofre ocorrem reações complexas que levam à formação de ligações cruzadas do tipo: C-S-C (monossulfídica), C-S $-\mathrm{C}$ (dissulfídica) ou C-S $-\mathrm{C}$ (polissulfídica). A densidade e o tipo de ligações cruzadas são parâmetros muito importantes devido aos seus efeitos dominantes sobre as propriedades mecânicas do composto ${ }^{[2]}$.

O tipo de ligação formada depende do sistema de vulcanização utilizado, do tempo e da temperatura de vulcanização. Dependendo da razão acelerador/enxofre presente na formulação, os sistemas de vulcanização podem ser classificados como convencional (CONV), eficiente (EV) e semi-eficiente (SEV). Cada um desses sistemas apresenta proporções distintas de ligações sulfídicas. O sistema convencional é caracterizado por baixa razão de acelerador-enxofre, resultando em alta formação de ligações polissulfídicas e estruturas ciclicas. O EV apresenta alta razão de acelerador-enxofre e no SEV o valor dessa razão é intermediario entre o CONV e EV. Quanto maior a razão 
acelerador-enxofre maior é a proporção de ligações mono e dissulfídicas ${ }^{[3]}$. Vulcanizados contendo predominantemente ligações monossulfídicas tem melhor resistência ao calor e a reversão, devido à estabilidade da ligação C-S. Já vulcanizados com maior teor de ligações polissulfídicas possuem maior resistência à tração e fadiga, devido a habilidade de ligações $\mathrm{S}-\mathrm{S}$ se quebrarem e se reorganizarem, aliviando altas tensões antes que se inicie a falha no material ${ }^{[4]}$.

A reometria é uma das técnicas mais utilizadas para monitoramento da formação de ligações cruzadas em borrachas. Durante o processo de formação dessas ligações, verifica-se que o aumento do torque na curva de vulcanização é constante até atingir um plateau, indicando que o processo de vulcanização está completo e que uma rede estável foi formada. Durante um prolongado tempo de aquecimento, ou altas temperaturas, o torque passa por um máximo e diminui, fenômeno esse conhecido como reversã ${ }^{[5]}$. A reversão ocorre pela cisão de cadeias ou ligações sulfídicas, uma dessulfurização que ocorre lentamente durante a vulcanização. As ligações polissulfídicas são mais instáveis que as dissulfídicas, e ambas são transformadas em monossulfídicas. A reversão também pode ser causada por uma despolimerização da cadeia polimérica. Esse fenômeno leva a uma redução das ligações cruzadas e das propriedades físicas do material ${ }^{[6]}$.

A influência da densidade e tipo de ligações cruzadas, nas propriedades mecânicas da borracha foi estudada por diversos autores ${ }^{[3,6,7]}$. No entanto, existe uma lacuna na literatura sobre a influência do método de vulcanização, na formação de ligações cruzadas e, consequentemente, nas propriedades mecânicas da borracha. Um desafio defrontado na fabricação de artefatos de borracha tem sido correlacionar o tempo ótimo de vulcanização $\left(t_{90}\right)$ fornecido pelo reômetro, que é usado na fabricação de artefatos prensados, para situações onde é necessário fabricar artefatos vulcanizados em autoclaves.

Nesse estudo avaliou-se a influência do método de vulcanização e da pressão utilizada nas propriedades: resistência ao rasgo, resistência à tração, densidade de ligações cruzadas total e ligações polissulfídicas, em uma formulação denominada NR4010-A, à base de borracha $\mathrm{NR}$, utilizando-se o sistema de vulcanização semi-eficiente.

\section{Materiais e Métodos}

\subsection{Componentes da formulação}

Para a fabricação das formulações à base de borracha NR, foram utilizados, em phr: (NR) GEB1 (100); peptizante ACTPLASS $(1,5)$; óxido de zinco $(\mathrm{ZnO})(5,0)$; negro de fumo HF330 Carbot (10); óleo parafínico $(4,0)$; agente de processo STUKTOL WB $212(1,0)$; ácido esteárico $(1,0)$; enxofre (1,0); dissulfeto de tetrametiltiuram (TMTD) $(0,5)$; 2,2,4-trimetil-1,2-dihidroquinolina (TMQ)-(1,0); dissulfeto de 2,2-dibenzotiazil (MBTS) $(1,5)$. Todos os componentes de grau comercial, foram usados como recebidos.

\subsection{Processo de mistura}

A mistura elastomérica foi preparada de acordo com a norma ASTM D3182 ${ }^{[8]} \mathrm{e}$ a dispersão dos ingredientes foi realizada, utilizando misturador aberto Luxor BML 150, com temperatura dos rolos igual a $40{ }^{\circ} \mathrm{C}$. A adição dos ingredientes segue a ordem da formulação, reservando a adição dos aceleradores e do agente de vulcanização (TMTD, MBTS e S) para o final do processo.

\subsection{Determinação dos parâmetros de vulcanização}

Para essa formulação, foi escolhido o sistema de vulcanização semi-eficiente e a mistura foi analisada em reômetro de disco oscilatório (ODR), modelo R100S da Monsanto a $145^{\circ} \mathrm{C}$, com arco de $3^{\circ}$, segundo a norma ASTM D-2084 [9], para estudo das condições de vulcanização. A partir da curva reométrica, determinou-se o torque máximo $(\mathrm{MH})$, o torque mínimo (ML) e o torque que corresponde a $90 \%$ das ligações cruzadas formadas, que possibilitou a determinação do $t_{90}$, calculado pela Equação 1.

$$
\mathrm{M}_{90}=\mathrm{ML}+0,9 *(\mathrm{MH}-\mathrm{ML})
$$

Com os dados apresentados na curva reométrica, definiu-se a faixa de tempo para analisar a vulcanização por prensagem e em autoclave, sem pressão.

\subsection{Vulcanização em prensa}

$\mathrm{Na}$ vulcanização por prensagem, a borracha crua foi disposta na cavidade do molde pré-aquecido a $145{ }^{\circ} \mathrm{C}$, sendo prensada por meio de uma prensa modelo 2532 Luxor com aquecimento elétrico, a temperatura de $145^{\circ} \mathrm{C}$ e pressão de $150 \mathrm{kgf} / \mathrm{cm}^{2}$, em períodos pré-definidos de 07, 10, 20, 45 e $90 \mathrm{~min}$. O molde utilizado para preparo de mantas com $2 \mathrm{~mm}$ de espessura segue a Norma ASTM D3182 ${ }^{[8]}$, de onde são retirados cdp's para os ensaios mecânicos. Também foi preparado um corpo de prova com 10 min de vulcanização e pressão de $10 \mathrm{kgf} / \mathrm{cm}^{2}$, para a comparação das propriedades mecânicas com material obtido com pressão de $150 \mathrm{kgf} / \mathrm{cm}^{2}$.

\subsection{Vulcanização por autoclave}

A borracha NR crua foi calandrada, com aproximadamente $2 \mathrm{~mm}$ de espessura em uma calandra marca LUXOR, de 04 rolos com L invertido, e em seguida, o material foi disposto na cavidade do molde pré-aquecido a $100^{\circ} \mathrm{C}$, para reduzir a viscosidade e facilitar a dispersão da borracha na cavidade, e prensado até o fechamento do molde. O molde utilizado foi o definido na Norma ASTM D3182 ${ }^{[8]}$. Em seguida, o molde foi inserido em autoclave a temperatura e pressão ambiente, e iniciou-se o aquecimento até $145^{\circ} \mathrm{C}$, o que levou cerca de 25 minutos. Depois de atingida essa temperatura iniciou-se a contagem dos tempos para o estudo da vulcanização.

Para avaliar se a formação das ligações cruzadas poderia ter início durante o tempo de aquecimento em autoclave, foi preparado um corpo de prova nas mesmas condições anteriores, mantido em autoclave por 30 minutos, até a estabilização da temperatura em $145^{\circ} \mathrm{C}$. Esse corpo de prova foi submetido a ensaio reométrico, e pela curva de vulcanização observou-se que $o t_{90}$ foi o mesmo da borracha não submetida ao processo de aquecimento em autoclave, indicando que não houve formação de ligações cruzadas durante o aquecimento do sistema. Em função destes resultados, após a estabilização da temperatura em $145^{\circ} \mathrm{C}$, os corpos de prova preparados em autoclave foram vulcanizados a pressão ambiente nos mesmos períodos pré-definidos para o processo em prensa: 07, 10, 20, 45 e $90 \mathrm{~min}$. 


\subsection{Propriedades mecânicas}

Foram realizados ensaios de resistência à tração ASTM D 412- tipo " $\mathrm{C}$ " [10] e resistência ao rasgo ASTM D 624- tipo "C" ${ }^{[11]}$ Os ensaios de resistência à tração foram realizados em máquina de ensaios INSTRON 3367 e resistência ao rasgo em máquina de ensaios ZWICK 1474 à temperatura ambiente com velocidade de ensaio de $500 \mathrm{~mm} / \mathrm{min}$.

\subsection{Ensaio de inchamento}

Antes da realização dos ensaios de inchamento, foram realizadas extrações em Soxhlet para remoção de materiais solúveis nas amostras vulcanizadas ${ }^{[12]}$. Iniciou-se a extração com acetona por $24 \mathrm{~h}$ a temperatura ambiente, para remoção de substâncias polares, tais como ativadores e aceleradores. Em seguida, realizou-se extração com ciclohexano, nas mesmas condições anteriores para remoção de substâncias apolares, como óleo e polímero não reticulado.

Após remoção das substâncias polares e apolares, a resistência ao inchamento das composições vulcanizadas de borracha natural foi avaliada pela imersão de corpos de prova pesando aproximadamente $0,25 \mathrm{~g}$ em $100 \mathrm{~mL}$ de ciclohexano. $\mathrm{O}$ ensaio foi realizado a temperatura ambiente e após 03 dias, as variações de massa se tornaram pouco significativas atingindo-se o equilíbrio, em seguida as amostras foram secas em estufa por $4 \mathrm{~h} \mathrm{a} 60^{\circ} \mathrm{C}$. A variação de massa foi medida desde a imersão no solvente até a retirada da estufa. Foram utilizados três corpos de prova de cada material.

A densidade de ligações cruzadas das amostras foi determinada usando as Equações 2 e 3 de Flory-Rehner ${ }^{[13]}$.

$$
\begin{aligned}
& v_{2}=\left[1+\left(\frac{m_{e q}-m_{d}}{m_{d}}\right)\left(\frac{\rho_{2}}{\rho_{1}}\right)\right]^{-1} \\
& v=\frac{\ln \left(1-v_{2}\right)+v_{2}+x v_{2}^{2}}{v_{1}\left(\frac{v_{2}}{2}-v_{2}^{1 / 3}\right)}
\end{aligned}
$$

$v_{2}$ é a fração volumétrica da borracha inchada; $m_{E Q}$ é a massa do corpo de prova inchado; $m_{D}$ é a massa do corpo de prova seco após o inchamento; $\rho_{1}$ e $\rho_{2}$ são a densidade do ciclohexano $(0,774 \mathrm{~g} / \mathrm{mL})$ e gel seco, respectivamente. $v$ é a densidade de ligações cruzadas; $x$ é o parâmetro de interação borracha-solvente (NR-ciclohexano 0,399) ${ }^{[14]}$. $v_{1}$ é o volume molar do ciclohexano $\left(108,75 \mathrm{~cm}^{3} \cdot \mathrm{mol}^{-1}\right)$.

As equações de Flory-Rehner, descritas acima, são válidas para sistemas sem cargas. Para sistemas com carga deve ser utilizada a correção de Kraus ${ }^{[15]}$. Como nesse caso as formulações têm a mesma quantidade de carga, a densidade de ligações cruzadas pode ser comparada sem a correção de Kraus.

\subsection{Determinação da concentração de ligações polissulfídicas $\left(v_{\text {poli }}\right)$}

A distribuição de ligações polissulfídicas, foi determinada por meio de reação com tiol-amina ${ }^{[16]}$. Para o preparo da solução de reação de quebra de ligações polissulfídicas acrescentou-se 37,6 $\mathrm{mL}$ de propanotiol e 39,5 $\mathrm{mL}$ de piperidina bidestilada em um balão de $1 \mathrm{~L}$ e o volume foi completado com heptano. As amostras foram imersas em $100 \mathrm{~mL}$ dessa solução, em atmosfera inerte de nitrogênio grau analítico e temperatura ambiente por duas horas. A reação foi interrompida por meio de lavagem com éter de petróleo por uma hora nas mesmas condições do ensaio. O processo de lavagem foi repetido por quatro vezes. Após a reação, determinou-se a densidade de ligações cruzadas por inchamento. Por meio da subtração do total de ligações cruzadas pela densidade de ligações cruzadas após reações tiol-amina, obteve-se a concentração de ligações polissulfídicas.

\section{Resultados e Discussão}

A partir da curva reométrica obtida para a amostra NR4010-A, Figura 1, foi possível calcular o torque máximo, $\mathrm{MH}=51 \mathrm{dN} . \mathrm{m}$, o torque mínimo, $\mathrm{ML}=2 \mathrm{dN} . \mathrm{m}$ e o torque equivalente a $90 \%$ de seu valor máximo, $M_{90}=46 \mathrm{dN}$.m e, consequentemente, o tempo de vulcanização, $\mathrm{t}_{90}=7 \mathrm{~min}$, equivalente a $\mathrm{M}_{90}$. Até o final do ensaio, ou seja, após 24 minutos, o material manteve suas propriedades reológicas inalteradas, não apresentando características de envelhecimento, que é consequência da cisão das cadeias poliméricas expostas a longos períodos sob aquecimento e cisalhamento. Com essa avaliação, foram definidos os períodos de 7, 10, 20, 45 e 90 minutos. Os tempos acima de 20 minutos tinham o propósito de simular o envelhecimento desse material, para os tempos de vulcanização em prensa e autoclave.

Na Figura 2 são apresentados os resultados das densidades de ligações cruzadas totais e ligações polissulfídicas das amostras vulcanizadas em prensa e em autoclave, nas mesmas condições de tempo e de temperatura de vulcanização. Comparando-se os resultados apresentados na Figura 2a, observa-se que o valor máximo de densidade total de ligações cruzadas para o material vulcanizado em prensa foi obtido em 7 minutos de vulcanização, correspondendo ao tempo ideal para vulcanização, conforme obtido em análise reométrica. A partir de 7 minutos observa-se uma redução acentuada da densidade de ligações cruzadas indicando que o processo de degradação da borracha tem início a partir de 7 minutos de processamento nas condições de preparação do material. O material vulcanizado em autoclave apresentou a quantidade máxima de ligações cruzadas em 10 min observando-se uma leve redução até

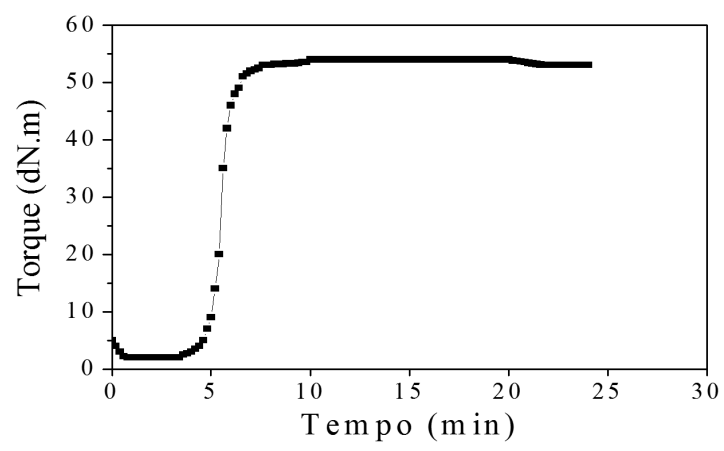

Figura 1. Curva reométrica da amostra NR4010-A. 
45 min de vulcanização. Em 90 minutos de vulcanização os valores de densidade de ligações cruzadas para os materiais obtidos pelos diferentes métodos são praticamente os mesmos. No entanto, em menores tempos de vulcanização, o material vulcanizado em autoclave apresenta valores de densidade de ligações cruzadas superiores aos verificados para o material preparado por prensagem.

A densidade de ligações polissulfídicas presentes na borracha obtida pelos diferentes métodos também foi avaliada. Segundo a literatura ${ }^{[12,16,17]}$, nos estágios iniciais da vulcanização ocorre uma maior formação de ligações polissulfídicas, $\mathrm{C}-\mathrm{S}_{\mathrm{x}}-\mathrm{C}(\mathrm{x}=4$ ou 5), que são dessulfurizadas, ou seja, ocorre uma diminuição do tamanho das ligações cruzadas levando a ligações monossulfídicas. Na Figura 2b é mostrada a variação na densidade de ligações polissufídicas em relação ao tempo e ao método de vulcanização da borracha natural. A redução na densidade de ligações polissulfídicas ocorre a partir de 07 min de vulcanização em prensa, coincidindo com a redução de densidade total de ligações cruzadas. Essa redução contínua deve-se, provavelmente, ao processo de reversão, proveniente da dessulfurização das ligações polissulfídicas ${ }^{[12,16,17]}$. O mesmo fenômeno é observado para a borracha vulcanizada em autoclave, porém numa intensidade menor.

Quando se comparam os métodos de vulcanização em prensa e em autoclave, tanto a transferência de calor que ocorre em cada método como os valores de pressão aplicados são condições que podem influenciar o tipo de ligações presentes e a formação de ligações cruzadas durante o processo. As variações observadas nos valores de densidade total de ligações cruzadas e densidade de ligações polissulfídicas podem ser explicadas considerando-se inicialmente o efeito de transferência calor que ocorre em cada método. Assumiu-se que os principais processos de transferência de calor que ocorrem nos diferentes métodos serão transferência de calor por condução ou por convecção para processamento em prensa ou autoclave, respectivamente. A transferência de calor por condução, que ocorre no processo por prensagem, é mais rápida favorecendo a formação de ligações polissulfídicas em 7 minutos de vulcanização, quando comparada à transferência de calor por convecção, na qual o máximo valor de ligações polissulfídicas foi obtido em

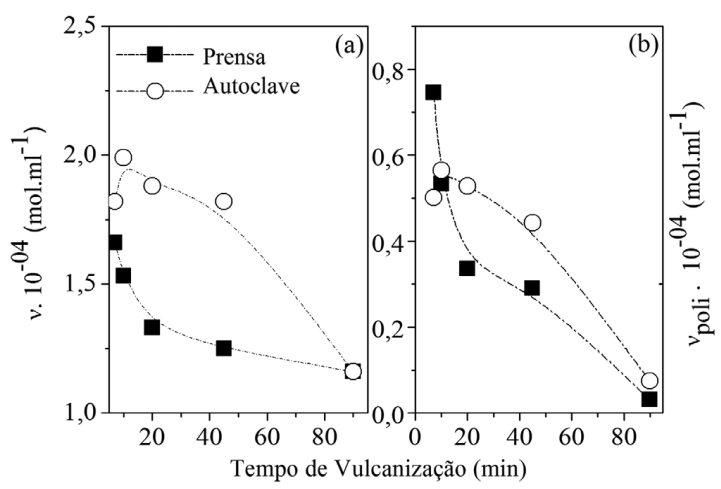

Figura 2. Variação da densidade de ligações cruzadas, com o tempo de vulcanização, em prensa e em autoclave: (a) ligações cruzadas totais e (b) ligações polissulfídicas.
10 minutos de vulcanização. As amostras submetidas à ação do calor por condução tem seu processo de envelhecimento acelerado em relação à transferência de calor por convecção, como no caso da autoclave, que se mostrou mais eficiente na formação de ligações cruzadas. Na autoclave, o calor é transferido de uma forma mais lenta, permitindo que as ligações polissulfídicas se quebrem e se rearranjem ${ }^{[18,19]}$, conservando tanto a densidade total de ligações cruzadas como a densidade de ligações polissulfídicas praticamente constantes até 45 minutos de vulcanização e prolongando o tempo para que se inicie o processo de degradação.

Além do efeito de transferência de calor na densidade de ligações cruzadas, obtidas pelos diferentes métodos, avaliou-se também a influência da pressão na vulcanização em prensa, comparando-se amostras vulcanizadas nas mesmas condições de tempo e temperatura, 10 minutos e $145^{\circ} \mathrm{C}$, a diferentes pressões, $10 \mathrm{Kgf.cm}{ }^{-2}$ e $150 \mathrm{Kgf.cm}{ }^{-2}$. Essas amostras foram submetidas a ensaios de inchamento e mecânicos, mostrados na Tabela 1.

A pressão também influencia a densidade total de ligações cruzadas e ligações polissulfídicas, observando-se que ambas foram reduzidas em menor pressão. Essa redução na densidade de ligações cruzadas foi explicado por Bellander ${ }^{[19]}$, que descreve que, com o aumento da pressão ocorre uma redução da mobilidade da cadeia polimérica, levando à diminuição do volume livre entre as moléculas. Essa maior proximidade entre as cadeias favorece a ocorrência das ligações entre o enxofre e as ligações insaturadas presentes em cadeias vizinhas, resultando em aumento da densidade de ligações cruzadas.

Para comparação do efeito da pressão em autoclave, além do material preparado a pressão ambiente, foi feita a vulcanização da borracha natural nas mesmas condições de tempo e temperatura, com pressão de $5 \mathrm{kgf} / \mathrm{cm}^{2}$, máximo valor de pressão disponível no equipamento utilizado. Os materiais preparados nessas duas condições de pressão foram comparados em relação à densidade de ligações cruzadas, à resistência à tração e ao alongamento, não sendo observadas variações nessas propriedades para os materiais obtidos em diferentes pressões, por esse motivo estes resultados não serão mostrados. Acredita-se que a aplicação deste nível de pressão não foi suficiente para causar alteração no processo de vulcanização por autoclave em relação à pressão ambiente.

A resistência ao rasgo da borracha NR obtida pelos diferentes métodos é mostrada na Figura 3, notando-se que os materiais vulcanizados por prensagem atingem a máxima resistência ao rasgo em 07 min de vulcanização e os vulcanizados em autoclave, em 10 min de vulcanização. A resistência ao rasgo para ambas às amostras é reduzida após 10 min de vulcanização. A maior resistência ao rasgo nesses tempos de vulcanização é coincidente com a maior

Tabela 1. Efeito da pressão $(\mathrm{P})$ na densidade total de ligações cruzadas $(v)$ e de ligações polissulfídicas $\left(v_{\text {oli }}\right)$ em borracha natural.

\begin{tabular}{|c|c|c|}
\hline $\begin{array}{c} \\
\left.(\mathrm{kgf.cm})^{-2}\right)\end{array}$ & $\begin{array}{c}\mathrm{v} \mathrm{10^{-4 }} \\
(\mathrm{mol} / \mathrm{mL})\end{array}$ & $\begin{array}{c}v_{\text {poli }} 10^{-4} \\
(\mathrm{~mol} / \mathrm{mL})\end{array}$ \\
\hline 150 & $1,53 \pm 0,02$ & $0,53 \pm 0,02$ \\
\hline 10 & $1,14 \pm 0,02$ & $0,40 \pm 0,02$ \\
\hline
\end{tabular}




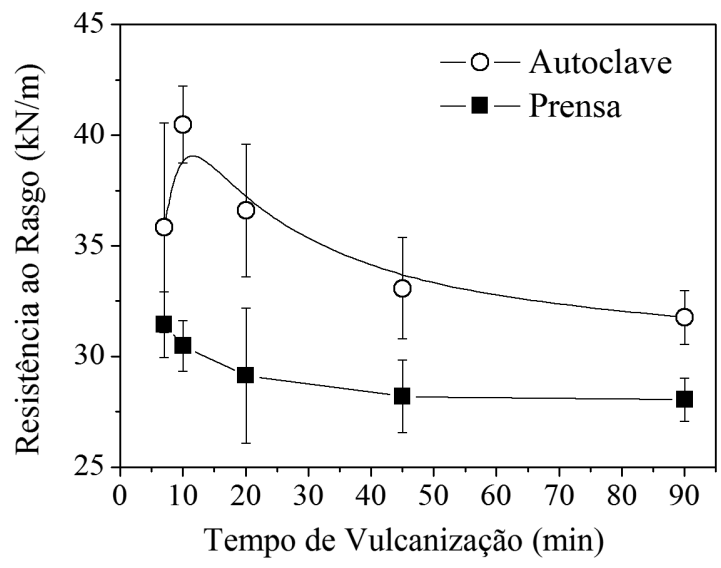

Figura 3. Variação da resistência ao rasgo em função do tempo de vulcanização.

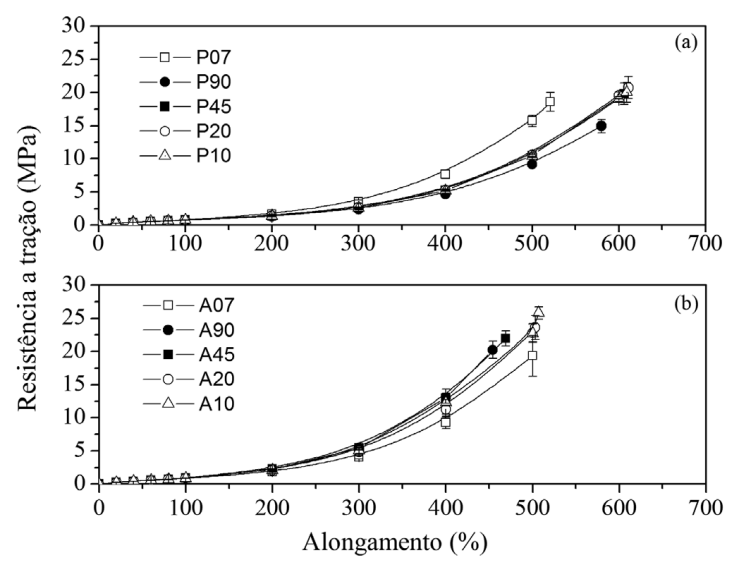

Figura 4. (a) Resistência à tração da NR4010 vulcanizada em prensa (P) versus alongamento, nos tempos (7, 10, 20, 45 e 90 minutos); (b) Resistência à tração da NR4010 vulcanizada em autoclave (A) versus alongamento nos tempos (7, 10, 20, 45 e 90 minutos).

densidade total de ligações cruzadas obtidas pelos diferentes métodos. Os valores de resistência ao rasgo são maiores para os materiais obtidos por autoclave em todos os tempos de vulcanização avaliados em relação aos materiais obtidos por prensagem. Essa propriedade aumenta com o aumento da densidade de ligações cruzadas, mas existe uma limitação que é o enfraquecimento causado pelo enrijecimento para altas densidades de ligação cruzada. A flexibilidade das ligações polissulfidicas é essencial para compensar esse enfraquecimento reduzindo a ocorrência de concentrações de tensão $0^{[16,18,20]}$. Assim, a maior resistência ao rasgo observada na borracha natural preparada por autoclave deve estar associada tanto a maior densidade de ligações cruzadas total como a maior densidade de ligações polissulfídicas presentes nesse material.

Os resultados dos ensaios de tração obtidos para a borracha NR4010-A vulcanizada por autoclave (Figura 4a) e por prensa (Figura $4 \mathrm{~b}$ ) mostram que o material preparado em autoclave apresentou uma resistência à tração $20 \%$ maior e alongamento na ruptura $30 \%$ menor em relação aos materiais obtidos por prensagem. Esses resultados podem ser justificados pela existência de uma maior densidade total de ligações cruzadas no material vulcanizado em autoclave. $\mathrm{O}$ aumento na densidade de ligações cruzadas atua no sentido de aumentar a energia necessária para a deformação e reduzir o alongamento pela diminuição do comprimento das cadeias poliméricas entre os pontos de ligação cruzada. Deve-se considerar ainda que acima de $300 \%$ de alongamento, a borracha natural cristaliza, influenciando diretamente sua resistência à tração ${ }^{[20,21]}$.

\section{Conclusão}

Os métodos de vulcanização empregados influenciaram o tipo e a concentração de ligações cruzadas em borracha natural, o que se refletiu em suas propriedades mecânicas. A influência dos diferentes métodos foi abordada em relação ao tipo de transferência de calor predominante e ao efeito da pressão aplicada durante a vulcanização. O material vulcanizado em autoclave apresentou maior densidade total de ligações cruzadas e ligações polissulfídicas, apresentando envelhecimento em maiores tempos de processamento (90 minutos), e melhores propriedades mecânicas quando comparado ao método por prensagem. Este resultado foi atribuído ao processo de transferência de calor por convecção, predominante no método de vulcanização em autoclave. $\mathrm{O}$ efeito da pressão não pode ser determinado na vulcanização por autoclave, obtendo-se materiais com propriedades similares, o que foi atribuído à pequena variação de pressão aplicada nesse caso. A borracha natural vulcanizada por prensagem em diferentes pressões apresentou variação na densidade total de ligações cruzadas e de ligações polissulfídicas, sendo estes parâmetros superiores para o material vulcanizado em maior pressão.

\section{Referências}

1. Hertz, D. L. (1984). Theory and Practice of vulcanization. Elastomerics, 116(11), 17-21.

2. Fan, R., Zhang, Y., Huang, C., Zhang, Y., Fan, Y., \& Sun, K. (2001). Effect of Crosslink Structures on Dynamic Mechanical Properties of Natural Rubber Vulcanizates under Different Aging Conditions. Journal of Applied Polymer Science, 81(3), 710-718. http://dx.doi.org/10.1002/app.1488.

3. Oliveira, M. G., \& Soares, B. G. (2002). Influência do sistema de vulcanização nas propriedades da mistura NBR/EPDM. Polimeros: Ciência e Tecnologia, 12(1), 11-19. http://dx.doi. org/10.1590/S0104-14282002000100007.

4. Hamed, G. R. (1992). Materials and compound. In: A. N. Gent (Ed.), Engeneering with rubber, how to design rubber components (pp. 21). New York: Hanser Publisher.

5. Mukhopadhyay, R., De, S. K., \& Chakraborty, S. (1977). Effect of vulcanization temperature and vulcanization systems on the structure and properties of natural rubber vulcanizates. Polymer, 18(12), 1243-1249. http://dx.doi.org/10.1016/00323861(77)90287-7.

6. Kok, C. M. (1987). The Effects of Compounding variables on the reversion process in the sulfhur vulcanization of natural rubber. European Polymer Journal, 23(8), 611-615. http:// dx.doi.org/10.1016/0014-3057(87)90006-1.

7. Nasir, M., \& Teh, G. K. (1988). The effects of various Types of Crosslinks on the Phisical Properties of Natural Rubber. 
European Polymer Journal, 24(8), 733-736. http://dx.doi. org/10.1016/0014-3057(88)90007-9.

8. American Society for Testing and Materials-ASTM. (2012). ASTM D3182: standard practice for rubber materials. equipment, and procedures for mixing standard compounds and preparing standard vulcanized sheets. West Conshohocken: ASTM.

9. American Society for Testing and Materials - ASTM. (2007). ASTM D-2084: standard practice for rubber materials vulcanization using oscillating disk cure. West Conshohocken: ASTM.

10. American Society for Testing and Materials-ASTM. (2012). ASTM D412 tipo C: rubber and thermoplastic elastomerstension. West Conshohocken: ASTM.

11. American Society for Testing and Materials -ASTM. (2007). ASTM D624 tipo C: standard test method for tear strength of conventional vulcanized rubber and thermoplastic elastomers. West Conshohocken: ASTM.

12. Dijkhuis, K. A. J., Noordermeer, J. W. M., \& Dierkes, W. K. (2009). The relationship between crosslink system, network structure and material properties of carbon black reinforced EPDM. European Polymer Journal, 45(11), 3302-3312. http:// dx.doi.org/10.1016/j.eurpolymj.2009.06.029.

13. Flory, P. J., \& Rehner, J. Jr (1943). Statistical mechanics of cross-linked polymer networks. The Journal of Chemical Physics, 11(11), 512-530. http://dx.doi.org/10.1063/1.1723791.

14. Aston, J. G., Szasz, G. J., \& Fink, H. L. (1943). The heat capacity and entropy, heats of transition, fusion and vaporization and vapor pressures of ciclohexane: the vibrational frequencies of alicyclic ring systems. Journal of the American Chemical Society, 65(6), 1135-1139.
15. Kraus, G. (1963). Swelling of filler-reinforced vulcanizates. Journal of Applied Polymer Science, 7(3), 861-871. http:// dx.doi.org/10.1002/app.1963.070070306.

16. Saville, B., \& Watson, A. A. (1967). Structural characterization of sulfur-vulcanized rubber networks. Rubber Chemistry and Technology, 40(1), 100-148. http://dx.doi.org/10.5254/1.3539039.

17. Mark, J. E., Burak, B., \& Frederick, R. E. (2005). Science and technology of rubber. Califórnia: Elsevier Academic Press.

18. Hamed, G. R. (2004). Tear strength of black filled natural rubber crosslinked via convencional and efficient sulfur cures. Rubber Chemistry and Technology, 77(2), 227-229. http:// dx.doi.org/10.5254/1.3547819.

19. Bellanger, M. (1998). High pressure vulcanization, crosslink of diene rubbers without vulcanization agents (Dissertação de mestrado). Royal Institute of Technology, Stockholm.

20. Chenal, J. M., Gauthier, C., Chazeau, L., Guy, L., \& Bomal, Y. (2007). Parameters governing strain induced crystallization in filled natural rubber. Polymer, 48(23), 6893-6902. http:// dx.doi.org/10.1016/j.polymer.2007.09.023.

21. Kok, C. M., \& Yee, V. H. (1986). The effects of crosslink density and crosslink type on the tensile and tear atrengths of NR, SBR and EPDM gum vulcanizates. European Polymer Journal, 22(4), 341-345. http://dx.doi.org/10.1016/00143057(86)90203-X.

Enviado: Jan. 15, 2015

Revisado: Maio 25, 2015

Aceito: Jun. 19, 2015 